\title{
A concise review on THGEM detectors
}

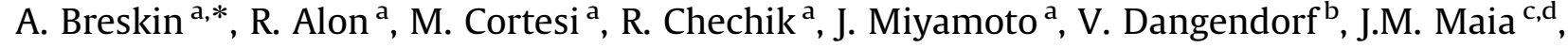 \\ J.M.F. Dos Santos ${ }^{c}$ \\ ${ }^{a}$ Department of Particle Physics, The Weizmann Institute of Science, 76100 Rehovot, Israel \\ ${ }^{\mathrm{b}}$ Physikalisch-Technische Bundesanstalt (PTB), Braunschweig, Germany \\ c Physics Department, University of Coimbra, 3004-516 Coimbra, Portugal \\ ${ }^{\mathrm{d}}$ University of Beira Interior, 6201-001 Covilhä, Portugal
}

\section{A R T I C L E I N F O}

Available online 22 August 2008

Keywords:

Gaseous electron multipliers THGEM

GEM

Radiation-imaging detectors

Gas avalanche multiplication

Hole multiplication

UV-photon detectors

Cryogenic gaseous detectors

\begin{abstract}
A B S T R A C T
We briefly review the concept and properties of the THick Gaseous Electron Multiplier (THGEM); it is a robust, high-gain gaseous electron multiplier, manufactured economically by standard printed-circuit drilling and etching technology. Its operation and structure resemble that of gaseous electron multiplier's (GEM's) but with 5-20-fold expanded dimensions. The millimeter-scale hole-size results in good electron transport and in large avalanche-multiplication factors, e.g. reaching $10^{7}$ in doubleTHGEM cascaded single-photoelectron detectors. The multiplier's material, parameters and shape can be application-tailored; it can operate practically in any counting gas, including noble gases, over a pressure range spanning from 1 mbar to several bars; its operation at cryogenic (LAr) conditions was recently demonstrated. The high gain, sub-millimeter spatial resolution, high counting-rate capability, good timing properties and the possibility of industrial production capability of large-area robust detectors, pave ways towards a broad spectrum of potential applications; some are discussed here in brief.
\end{abstract}

(c) 2008 Elsevier B.V. All rights reserved.

\section{Introduction}

Gaseous avalanche radiation-imaging detectors have been subject to intensive developments over the past decades. The so-called micropattern detectors, produced by different microlithographic techniques provide localization resolutions in the few-tens of micrometers range, approaching that of silicon trackers [1]. The most advanced operative micropattern detectors are cascaded gaseous electron multipliers (GEM) [2,3] and the Micromegas [4,5]. Within the broad family of micropattern gas detectors, the THick GEM (THGEM) is one of the most recent developments [6]; it is attracting significant attention due to its simplicity and robustness. The THGEM has a hole-structure similar to the GEM, but with about 5-20-fold expanded dimensions (Fig. 1). It is manufactured economically by mechanically drilling sub-millimeter diameter $(d)$ holes, spaced by a fraction of a $\mathrm{mm}(a)$ in a thin ( $t$, generally a fraction of a $\mathrm{mm}$ ) printed-circuit board (PCB), followed by Cu-etching of the hole's rim (typically $0.1 \mathrm{~mm}$ ). In addition to the standard etching using photolithographic masks (e.g. our THGEMs were manufactured by this process by Print Electronics Inc., Israel. www.print-e.co.il), a simpler mask-less etching technique was recently proposed and is

\footnotetext{
* Corresponding author. Tel.: +972 89342645 ; fax: +972 89342611

E-mail address: amos.breskin@weizmann.ac.il (A. Breskin).
}

under investigations [7]. The etched rim reduces edge discharges, resulting in over 10 -fold higher gains than without a rim (Fig. 2); e.g. the "optimized GEM" [8] or "LEM" [9] have no rims.

Two or more THGEM elements can be cascaded, to provide higher gains or increase operation stability. THGEMs may be fabricated out of various PCB materials-e.g. FR-4, G-10, Kevlar, Cirlex (polyimide with low natural radioactivity [10]), Teflon, etc. Due to their mechanical robustness, THGEM-based detectors may be constructed with very large area and their implementation does not require any particular mechanical supports.

In this work we briefly review the operation principle of THGEM detectors and their properties. Details can be found in previous articles [11-15] and theses [16,17]. Some recent results on time resolution and operation in noble gases as well as potential applications are briefly discussed.

\section{THGEM operation and properties}

\subsection{General}

The THGEM's operation principle is basically the same as that of the GEM: an electric potential is applied between the electrodes and creates a strong dipole electric field within the holes, protruding also into the adjacent volume. This particular shape 


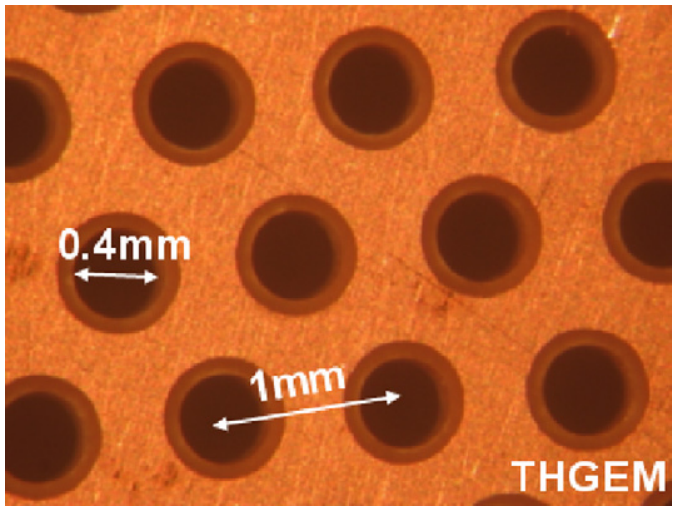

Fig. 1. Photograph of a typical THGEM electrode; the one shown has a holediameter of $d=0.4 \mathrm{~mm}$ with $0.1 \mathrm{~mm}$ etched rim, spaced by $a=1 \mathrm{~mm}$. The thickness is $t=0.5 \mathrm{~mm}$.

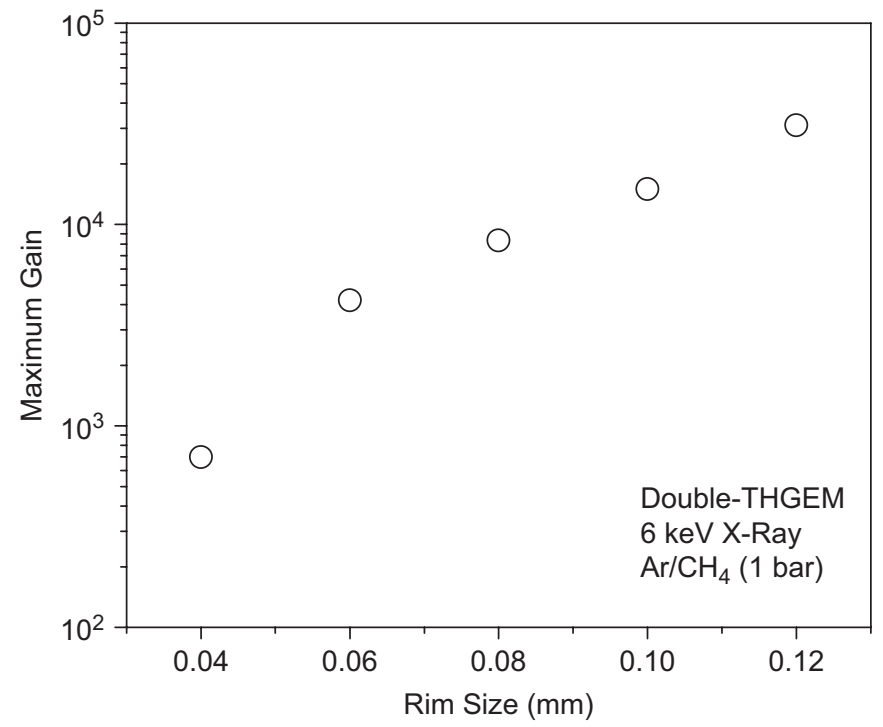

Fig. 2. Maximum attainable gain vs. rim size. Detector parameters: $t=0.4 \mathrm{~mm}$; $a=1 \mathrm{~mm} ; d=0.3 \mathrm{~mm}$.

of the field is responsible for an efficient focusing of ionization electrons into the holes and their multiplication by a gas avalanche process. The electron collection is more effective than in GEM because the THGEM's hole-diameter is larger than the electron's transverse diffusion range when approaching the hole. The efficient collection and transmission of electrons offers the possibility to use several THGEM elements in cascade. This leads to higher detector gains at lower voltage bias per single THGEM element and thus to higher operation stability. This is important when the detected radiation has a large dynamic range in primary ionization density (e.g. neutrons, radioactive background, etc.). The THGEM can efficiently detect radiation-induced electrons, either deposited in the gas or emitted from a solid converter. While the former is important for particle tracking, X-ray imaging (Fig. 3), etc., the latter has important applications in single-photon [13] and neutron imaging.

The results of systematic studies of THGEM-based detectors, operating at atmospheric and low gas pressures, have been extensively reported in Refs. [11,12]. The role of various geometrical and operational parameters, optimal conditions for reaching full single-photoelectron detection efficiency and maximal electron transport were established. The last two prerequisites are particularly important for applications necessitating efficient photon-counting and -imaging with solid photocathodes, as in

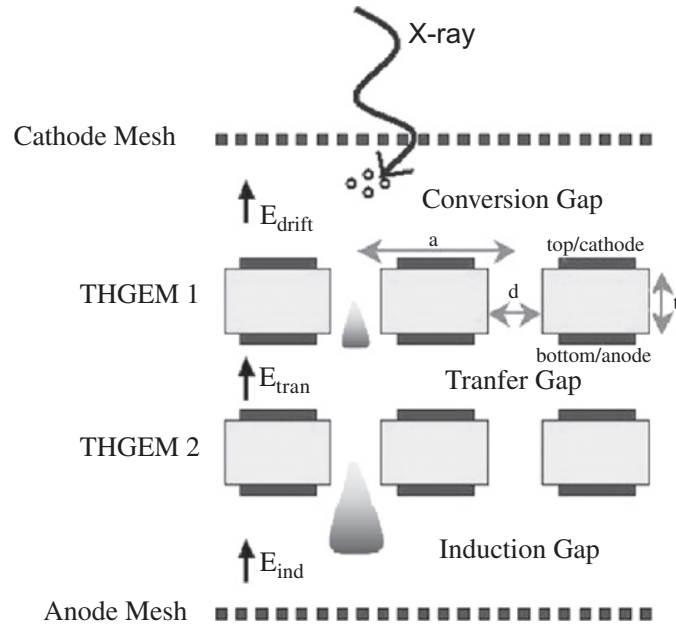

Fig. 3. Schematic view of a double-THGEM soft X-ray detector; the same configuration is adequate for particle tracking and timing.

a

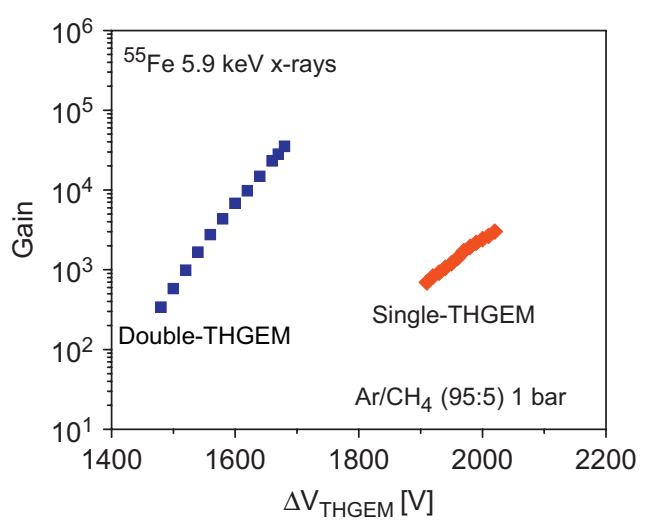

b

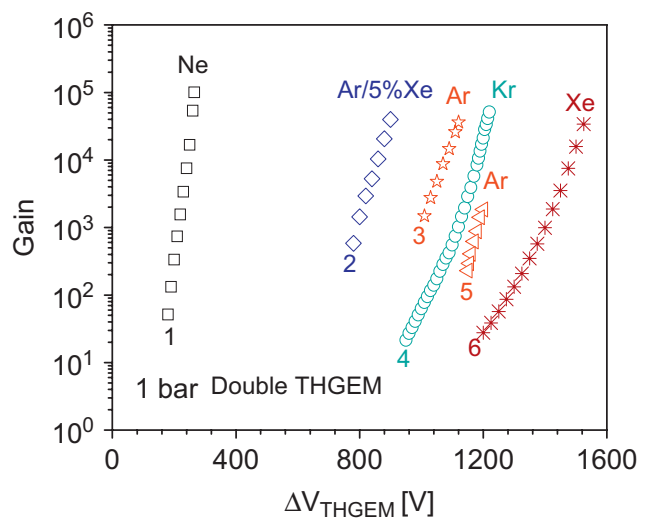

Fig. 4. Gain curves with $5.9 \mathrm{keV}$ X-rays. (a) In single- and double-THGEM ( $t=0.8 \mathrm{~mm}, d=0.6 \mathrm{~mm}, a=1 \mathrm{~mm}$ ) in $1 \mathrm{bar} \mathrm{Ar} / 5 \% \mathrm{CH}_{4}$ and (b) in double-THGEM in noble gases at 1 bar. In (a), except for Ar curve (3), measured in gas-flow mode (not purified), all other data were measured with getter-purified gases [18].

Cherenkov Ring Imaging detectors (RICH). It was found that due to the large hole size, efficient electron transport and negligible photon- and ion-feedback, the THGEM has stable operation in a large variety of gas mixtures, including noble gases. High attainable gains, $>10^{4}$ and $>10^{6}$, were reached with single photoelectrons in single- and in double-THGEM detectors, respectively, at $1 \mathrm{~atm}$ of $\mathrm{Ar} / 5 \% \mathrm{CH}_{4}$ and $\mathrm{Ar} / 30 \% \mathrm{CO}_{2}$, thus assuring good sensitivity for single-photon detection. [11,13]. The same 
detectors yielded gains of $>10^{3}$ and $>10^{4}$ in single- and doubleTHGEM arrangements, respectively, with few-hundred primary electrons induced by $5.9 \mathrm{keV} \mathrm{X}$-rays in $1 \mathrm{~atm} \mathrm{Ar} / 5 \% \mathrm{CH}_{4}$ [14] (Fig. 4a). In this gas the THGEM reached counting-rate capabilities $>1 \mathrm{MHz} / \mathrm{mm}^{2}$ at effective gains of $\sim 10^{4}[11]$.

The localization resolution was studied with a $10 \times 10 \mathrm{~cm}^{2}$ double-THGEM detector irradiated with $8 \mathrm{keV} X$-rays. It comprised two THGEM electrodes of $t=0.4 \mathrm{~mm}, d=0.5 \mathrm{~mm}$ and $a=1 \mathrm{~mm}$, coupled to a resistive anode; the latter broadened the induced signals, to match the $2 \mathrm{~mm}$ pitch of the $\mathrm{X}-\mathrm{Y}$ delay-line readout electrode placed behind it. Localization resolutions of $\sim 0.7 \mathrm{FWHM}$ (smaller than the hole-pitch) were reached in $1 \mathrm{bar} \mathrm{Ar} / 5 \% \mathrm{CH}_{4}$ at a gain of $10^{4}$; the gain variation was of $10 \%$ FWHM over the whole surface [14].

\subsection{Noble gases and low temperatures}

Gains above $10^{4}$ were recently measured at room temperature in a double-THGEM with $5.9 \mathrm{keV}$ X-rays in $1 \mathrm{bar} \mathrm{Ar}$, Kr, Xe, Ne and $\mathrm{Ar} / 5 \% \mathrm{Xe}$ (Fig. 4b) [18]; gains $>10^{3}$ were also reached in some of these gases at 2-3-fold higher pressures [18,19]. The energy resolution dependence on various parameters (gas type, pressure, electrode's geometry and electric fields) was studied in detail in noble gases, yielding in some configurations values below $20 \%$ FWHM for $5.9 \mathrm{keV}$ X-rays [18].

Recent studies indicated that double-THGEM detectors operated in two-phase liquid $\mathrm{Ar}$ could reach gains of $\sim 10^{4}$ [20]. The successful operation of THGEM detectors in cryogenic conditions was also reported in Refs. [21,22]. Slower signal development compared to that in cascaded-GEM multipliers was observed in the two-phase operation mode; it permitted noise reduction by pulse-shape analysis and thus lower detection thresholds [20].

\subsection{Rim effects and stability}

As discussed above and shown in Fig. 2, the size of the etched rim around the THGEM holes, is essential for reducing significantly discharge-occurrence probability; this permitted operation at higher permissible voltages and hence at higher detector gains. The relationship between maximum gain vs. rim-size was investigated with a $3 \times 3 \mathrm{~cm}^{2}$ double-THGEM, made from standard FR-4 PCB material, operating in $\mathrm{Ar} / 5 \% \mathrm{CH}_{4}$ at atmospheric pressure (setup shown in Fig. 3). It was irradiated with a collimated $\left(1 \mathrm{~mm}^{2}\right){ }^{55} \mathrm{Fe} \mathrm{X}$-ray beam. The maximum attainable gain was defined as the one at which micro-discharges were not observed for at least $20 \mathrm{~s}$. The maximum attainable gain increased practically exponentially with the rim-size (Fig. 2). This effect, as well as the gain stability in time, is due to a combination of several factors: electric field distribution outside the hole, charging up of the insulator, type of material, quality of hole's wall-surface, the surface-quality of the Cu-edge, etc. The charging up of the insulator also depends on gain and counting rate. Preliminary results indicated that gain-stabilization with time occurs within a few hours [15]. The matter is being thoroughly investigated in cooperation with CERN and INFN-Trieste within the CERN-RD51 collaboration.

\subsection{Time resolution}

The time resolution of a double-THGEM operating in 1 bar $\mathrm{Ar} /$ $5 \% \mathrm{CH}_{4}$ at room temperature was measured with UV photons (pulsed UV lamp) and with minimum ionizing charged particles (MIPs).

The detector assembled for UV-photon studies (Fig. 5) had a CsI photocathode, either evaporated on a transparent quartz plate installed at a $3 \mathrm{~mm}$ distance from the first THGEM (semitransparent photocathode), or evaporated directly onto the top surface of the first THGEM (reflective photocathode). Absorbers were used to adjust the number of incident photons per pulse. Both configurations yielded rather similar gain and time-resolution results [17].

Fig. 6 depicts the time resolution measured with a reflective photocathode (Fig. 5). It varied between 8 and $1 \mathrm{~ns}$ (RMS) for 1 to $\sim 100$ photoelectrons per UV-pulse. The time resolution for 1000photoelectron pulses was about 0.5 ns (RMS) [17].

The improved time-resolution with the number of photoelectrons results from measuring the "first-arriving photoelectron" (among those photo-produced at different locations on the photocathode's surface or arriving at different times due to diffusion [17]) and from improved signal-to-noise ratio.

Measurements with MIPs were done while converting radiation in a $3 \mathrm{~mm}$ drift gap (Fig. 3). The THGEM's time-pulses were measured against scintillators, either with ${ }^{106} \mathrm{Ru}$ beta-electrons or with cosmic rays. Both MIPs yielded similar time-resolution values (Fig. 7) of the order of $10 \mathrm{~ns}$ (RMS). The tail in the time distribution is due to the statistical pulse-height distribution of single ionization-electron pulses, affecting the trigger electronics. Note that we obtained, with the same setup and electronics, very similar tail-shape and resolution (7-8 ns RMS) were observed

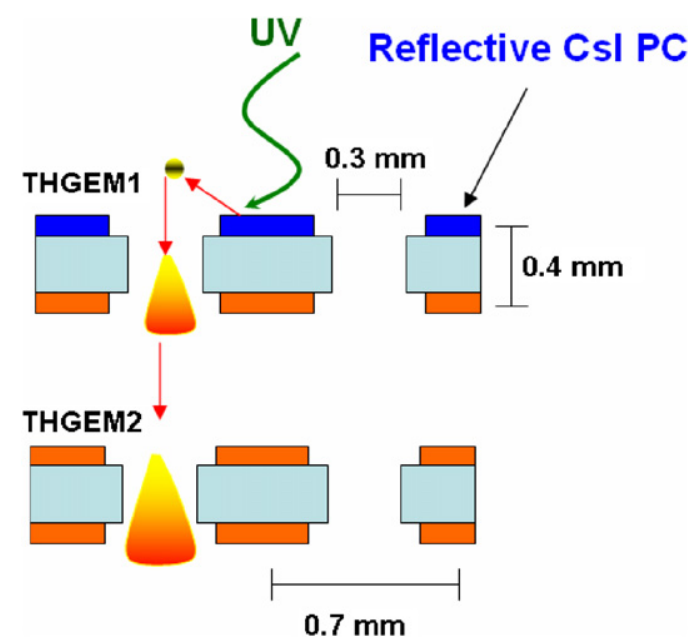

Fig. 5. Schematic view of a double-THGEM with a reflective CsI photocathode deposited on the top one. Photoelectrons are efficiently focused into THGEM1 and multiplied in two steps.

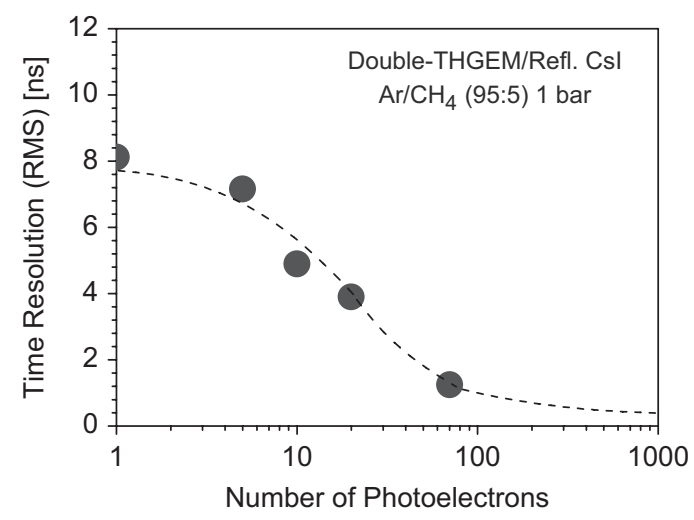

Fig. 6. Time resolution (RMS) vs. number of photoelectrons recorded with a pulsed UV lamp in a reflective double-THGEM gaseous photomultiplier with CsI photocathode of Fig. 5. 


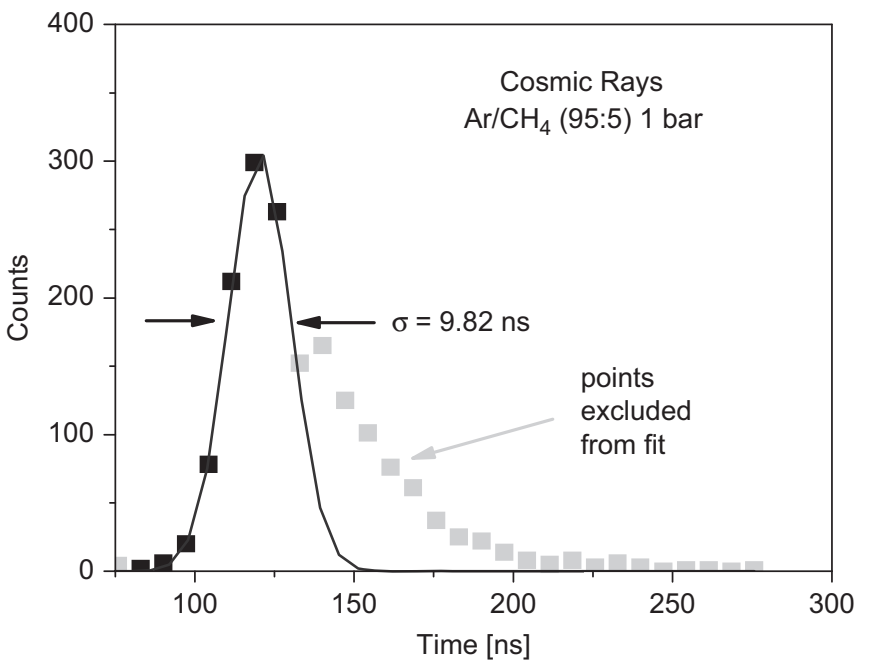

Fig. 7. Time resolution of a double THGEM (of Fig. 3) measured with cosmic rays.

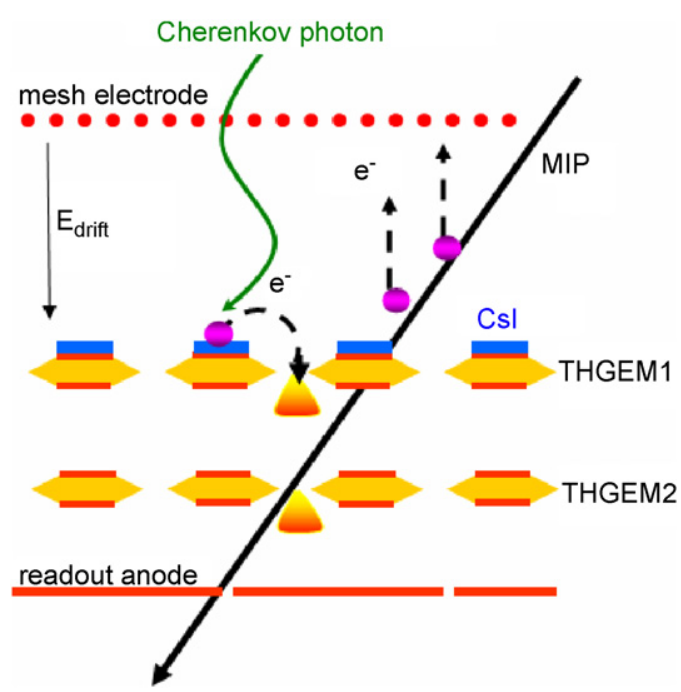

Fig. 8. A double-THGEM gaseous photon detector for RICH. The mesh defines a small reversed drift-field above the photocathode, repelling a major fraction of ionization electrons; the photoelectron collection efficiency is very little affected.

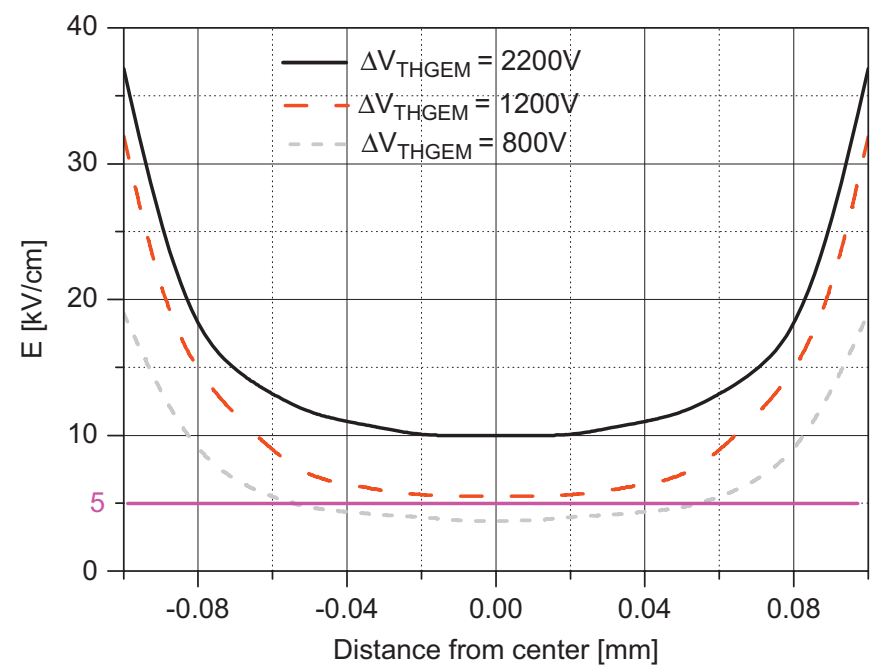

Fig. 9. Electric field on photocathode surface vs. distance from the center between two holes, created by the hole dipole field in the reflective-photocathode photon detector of Fig. 5 THGEM parameters: $d=0.3 \mathrm{~mm} ; a=0.7 \mathrm{~mm} ; t=0.4 \mathrm{~mm}$. with a standard triple-GEM detector. More details are given in Ref. [17].

\section{THGEM potential applications}

The robustness, simplicity and properties of the THGEM and the possibility of industrial production capability of large-area detectors, pave ways towards a broad spectrum of potential applications. These could rely on THGEM's single-electron sensitivity, moderate (sub-mm) localization resolution, timing in the $10 \mathrm{~ns}$ range, high-rate capability, low-temperature and broad pressure-range (mbar to few bar) operation.

Particle- and astroparticle-physics applications could encompass: tracking at moderate resolutions (e.g. large-area muon- or cosmic-ray detectors), sampling-elements for calorimeters, largevolume TPCs for rare events, single-photon detectors for RICH, etc.

a

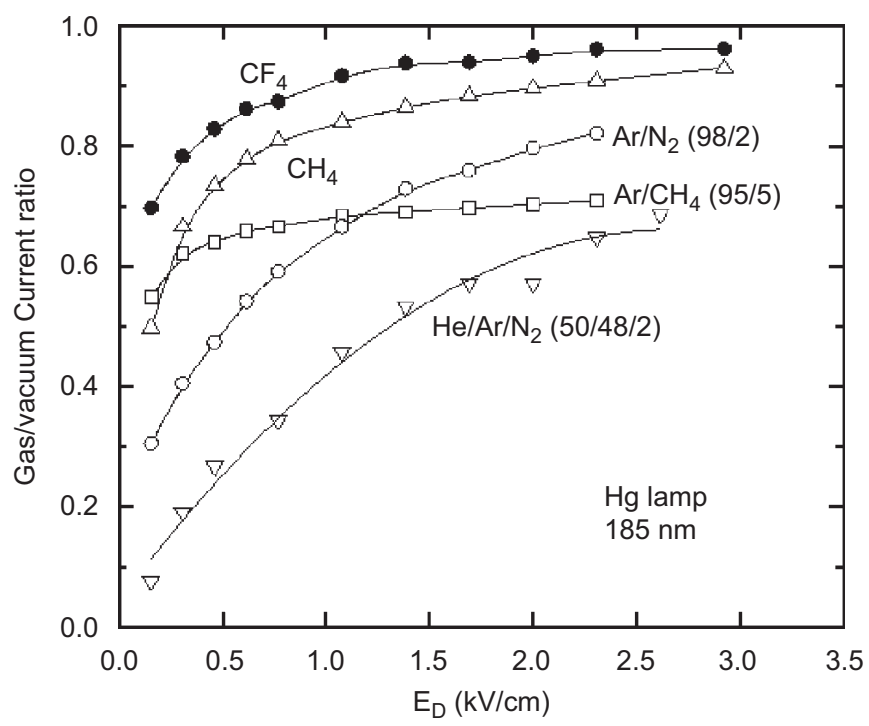

b

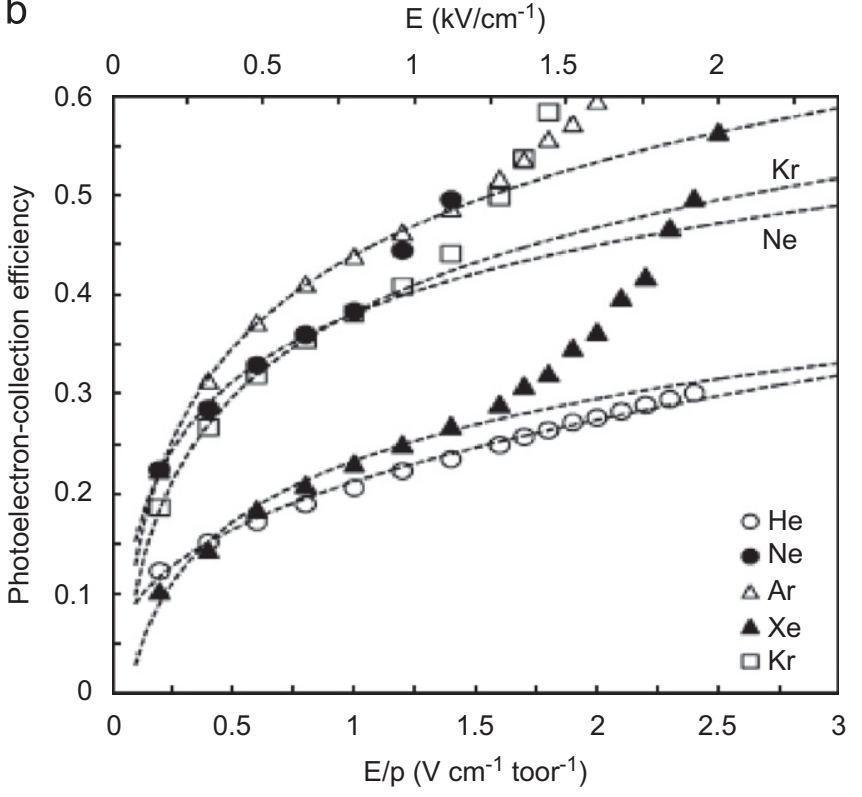

Fig. 10. Measured photoelectron collection efficiency into different gases vs. electric field in: (a) molecular gases and gas mixtures [26] and (b) noble gases. In the latter the dashed lines are model-calculated; the divergence measured in $\mathrm{Ar}$, $\mathrm{Kr}$, Ne and $\mathrm{Xe}$ for $E>1.2 \mathrm{kV} / \mathrm{cm}$ originates from gas excitation onset [27]. 


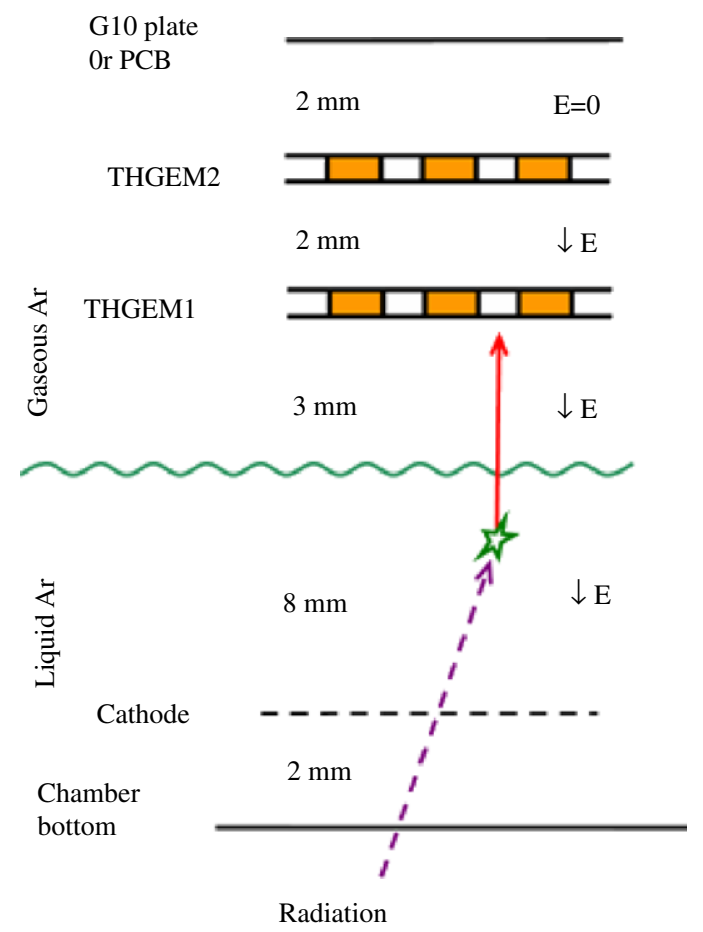

Fig. 11. Example of a double-THGEM cryogenic detector measuring radiationcharges extracted from noble-liquid. A photocathode deposited on the front face of THGEM1 (not shown) would also detect noble-liquid scintillation light [20].

Large-area THGEM UV-photon detectors with reflective CsI photocathodes [11,13] (Fig. 5) would have some advantages over cascaded-GEM ones [23,24]; e.g. the better electron collection and transport between cascaded elements results in a lower gain required at each single-multiplier step or, alternatively, fewer cascaded elements for an equal total gain. Like in reflective-GEM photomultipliers, a small reversed drift field above the photocathode reduces significantly the detector's sensitivity to charged particles (Fig. 8) [23,25].

The high electric fields at the THGEM's photocathode surface (between holes), reaching values of a few $\mathrm{kV} / \mathrm{cm}$, even at low THGEM potentials, (Fig. 9) assure good photoelectron extraction. The latter results in reasonably good effective-QE values [26] also in noble-gas mixtures (Fig. 10) [27,28].

The operation of THGEMs in noble gases has potential applications in gas-scintillation radiation detectors, two-phase (Fig. 11) and noble-liquid cryogenic detectors for rare events and for Gamma imaging. Cryogenic gaseous UV-photomultipliers with THGEM-coated CsI photocathodes are under development for scintillation-light recording in some of these applications. This should pave ways towards development of novel large-mass detectors, having high sensitivity to rare events, with lowradioactivity background (e.g. compared with photomultiplier tubes), and low-energy threshold-at moderate costs. Potential applications are in the fields of neutrino, double-beta decay and dark-matter physics.

Other fields in which THGEM detectors are under R\&D are: soft $\mathrm{X}$-ray imaging (Fig. 3), thermal- and fast-neutron imaging with adequate converters coupled to the multiplier or deposited on its surface (similar to Fig. 5), etc.

Last but not least is the resistance to damaging discharges, e.g. in cases of single-electron (photoelectron) or low-ionization (MIPs) detection accompanied occasionally by heavily ionizing background. Laboratory studies proved the THGEM to be robust and very resistant to sparks, compared to GEM.

A REsistive THGEM (RETHGEM) [28] was recently introduced, in an attempt to conceive a spark-immune multiplier. In the RETHGEM the Cu-clad (Fig. 1 ) is replaced by a resistive coating e.g. resistive Kapton, silk-screen-printed surface, etc. Like other detectors with resistive surfaces (e.g. RPCs) it has an improved resistance to discharges, but at the expense of lower counting-rate capability-of the order of $10-100 \mathrm{~Hz} / \mathrm{mm}^{2}$. Gains $\geqslant 10^{5}$ were reached in different gases in double-RETHGEM coupled to a CsI photocathode [28].

\section{Acknowledgements}

This work was partly supported by the Israel Science Foundation, Grant 402/05, the MINERVA Foundation Grant 8566 and the Foundation for Science and Technology (FCT)-Portugal, Project POCI/FP/81980/2007. We greatly acknowledge the assistance of V. Revivo and G. Genach, the PCB producers (Print Electronics, Israel, www.print-e.co.il). A. Breskin is the W.P. Reuther Professor of Research in The Peaceful Use of Atomic Energy.

\section{References}

[1] M. Titov, Nucl. Instr. and Meth. A 581 (2007) 24 (and refs. therein).

[2] F. Sauli, Nucl. Instr. and Meth. A 386 (1997) 531.

[3] F. Sauli, Nucl. Instr. and Meth. A 580 (2007) 971 (and refs. therein).

[4] Y. Giomataris, et al., Nucl. Instr. and Meth. A 376 (1996) 29.

[5] J. Bouchez, et al., Nucl. Instr. and Meth. A 574 (2007) 425 (and refs. therein).

[6] R. Chechik, et al., Nucl. Instr. and Meth. A 535 (2004) 303.

[7] Rui de Oliveira, MPGD technologies, in: Workshop on Micropattern Gas Detectors, CERN, 10-11 September 2007. 〈http://indico.cern.ch/ conferenceOtherViews.py?view=standard\&confld $=16213\rangle$.

[8] L. Periale, et al., Nucl. Instr. and Meth. A 478 (2002) 377.

[9] P. Jeanneret, Time projection chambers and detection of neutrinos, Ph.D. Thesis, Neuchâtel University, 2001.

[10] M. Gai, et al., arXiv:0706.1106.

[11] C. Shalem, et al., Nucl. Instr. and Meth. A 558 (2006) 475

[12] C. Shalem, et al., Nucl. Instr. and Meth. A 558 (2006) 468.

[13] R. Chechik, et al., Nucl. Instr. and Meth. A 553 (2005) 35.

[14] M. Cortesi et al., 2007 JINST 2 P09002.

[15] R. Chechik, et al. 〈http://www.slac.stanford.edu/econf/C0604032/papers/ 0025.PDF>.

[16] C. Shalem, MSc thesis, Weizmann Institute of Science. 〈http://jinst.sissa.it/ jinst/theses/2005_JINST_TH_001.pdf $>$.

[17] R. Alon, MSc thesis, Weizmann Institute of Science. 〈http://jinst.sissa.it/jinst/ theses/2008_JINST_TH_001.pdf $>$.

[18] R. Alon et al., 2008 JINST 3 P01005.

[19] J. Maia, et al., Characterization of the THGEM electron multiplier in Ne and Kr. In preparation.

[20] A. Bondar, et al., 2008_JINST_3_P07001.

[21] L. Periale, et al., Nucl. Instr. and Meth. A 567 (2006) 381.

[22] V. Peskov, et al., IEEE Trans. Nucl. Sci. NS54 (2007) 1784.

[23] D. Mörmann, et al., Nucl. Instr. and Meth. A 530 (2004) 258.

[24] Z. Fraenkel, et al., Nucl. Instr. and Meth. A 546 (2005) 466.

[25] S. Milov, et al., J. Phys. G: Nucl. Part. Phys. 34 (2007) S701.

[26] A. Breskin, et al., Nucl. Instr. and Meth. A 483 (2002) 670.

[27] L.C.C. Coelho, et al., Nucl. Instr. and Meth. A 581 (2007) 190 (and refs. therein).

[28] G. Agocs, et al., 2008 JINST 3 P02012 (and refs. therein). 